\title{
Remarks on BCOV invariants and degenerations of Calabi-Yau manifolds
}

\author{
Kefeng Liu and Wei Xia
}

\begin{abstract}
For a one parameter family of Calabi-Yau threefolds, Green, Griffiths and Kerr have expressed the total singularities in terms of the degrees of Hodge bundles and Euler number of the general fiber. In this paper, we show that the total singularities can be expressed by the sum of asymptotic values of BCOV invariants, studied by Fang, Lu and Yoshikawa. On the other hand, by using Yau's Schwarz lemma, we prove Arakelov type inequalities and Euler number bound for Calabi-Yau family over a compact Riemann surface.
\end{abstract}

\section{Introduction}

Reidemeister torsion (R-torsion) is an invariant that can distinguish between closed manifolds which are homotopy equivalent but not homeomorphic. Analytic torsion (or Ray-Singer torsion) is an invariant of Riemannian manifolds defined by Ray and Singer [RS71, RS73] as an analytic analogue of Reidemeister torsion. These two torsions naturally coincide, which is known as the Cheeger-Müller theorem [M $7 \ddot{8}$, Che79].

In [BCOV93, BCOV94], Bershadsky-Cecotti-Ooguri-Vafa conjectured an equivalence between the physical quantity $F_{1}$ of a Calabi-Yau threefold and a linear combination of the holomorphic analytic torsions, which now called the BCOV torsions. Motivated by their conjecture, Fang, Lu and Yoshikawa [FLY08] considered a modification of the BCOV torsions, called the BCOV invariants. They have conducted a detailed study of the asymptotic behavior of the BCOV invariants for the mirror quintic threefold. Recently, Yoshikawa [Yos14, Yos] has continued the study of the asymptotic behavior of the BCOV invariants for general degenerations of Calabi-Yau threefolds.

By using the curvature formula for Quillen metrics [BGS88a, BGS88b, BGS88c], Bershadsky-Cecotti-Ooguri-Vafa obtained a variational formula for the BCOV torsion of Ricci-flat Calabi-Yau manifolds. Fang-Lu [FL05] expressed the variation of the BCOV torsion of Ricci-flat Calabi-Yau manifolds as a linear combination of the WeilPetersson metric and the generalized Hodge metrics. Using this, they managed to show that for primitive (i.e. $\omega_{H^{k}}=0, \forall k<n$, see Section 2.2) Calabi-Yau manifolds of dimension $n$, if the Euler characteristic number satisfy $(-1)^{n} \chi<24$, then there exists no complete curve in $\mathcal{M}$, the moduli space of polarized Calabi-Yau manifolds.

In their paper [GGK09], Green, Griffiths and Kerr have studied global enumerative invariants of a polarized variation of Hodge structures for Calabi-Yau manifolds. By applying the Grothendieck-Riemann-Roch theorem, they can express the total singularities in terms of the degrees of Hodge bundles and Euler number of the general 
fiber:

$$
(\chi+36) \operatorname{deg} \mathcal{H}_{e}^{3,0}+12 \operatorname{deg} \mathcal{H}_{e}^{2,1}=2 \sum_{j} \Delta_{j}+\sum_{i}\left\{\chi_{2}^{i}-3 I_{2}^{i}\right\},
$$

where $\mathcal{H}_{e}^{3,0}, \mathcal{H}_{e}^{2,1}$ are the canonical Deligne's extensions of the Hodge bundles $\mathcal{H}^{3,0}, \mathcal{H}^{2,1}$, for each $j, \Delta_{j}$ is the number of ODP's on the singular fiber, and for each $i, \chi_{2}^{i}-3 I_{2}^{i}$ is the topological and intersecting data of the singular fiber. See (3.11) for precise definition. As a corollary, they showed that a non-degenerate family of Calabi-Yau threefolds must satisfy $\chi \leq-24^{1}$.

Let $\mathcal{X}$ be a smooth projective variety of dimension $n+1$ and let $S$ be a compact Riemann surface, $f: \mathcal{X} \rightarrow S$ be a surjective, flat holomorphic map with generic fiber Calabi-Yau $n$-fold, and $f^{0}: \mathcal{X}^{0} \rightarrow S^{0}$ be the smooth part of $f$. Unless stated otherwise we shall assume all the local monodromy transformations around points $s_{i} \in S \backslash S^{0}$ are unipotent. This is our basic assumption throughout this paper. By integrating the current equation due to Fang, Lu and Yoshikawa, we find that (for $n=3$ ) the total singularities can be expressed by the sum of asymptotic values of BCOV invariants:

Theorem 0.1. Let $E:=S \backslash S^{0}=\left\{s_{i}\right\} \subset S$ be the discriminant locus of $f$ and $\tau_{\mathrm{BCOV}}(t)$ be the BCOV invariant of the fiber $f^{-1}(t)$. Set

$$
a_{i}:=\lim _{t \rightarrow s_{i}} \frac{\log \tau_{\mathrm{BCOV}}(t)}{\log |t|^{2}} .
$$

Then

$$
(\chi+36) \operatorname{deg} \mathcal{H}_{e}^{3,0}+12 \operatorname{deg} \mathcal{H}_{e}^{2,1}=12 \sum_{i} a_{i} .
$$

The interesting feature of this identity is that the left hand side involves only the zero spectrum, or the harmonic space, of the Laplacian operator, while the right hand side is derived from the whole spectrum. As a direct corollary, we see that

\section{Corollary 0.2 .}

$$
12 \sum_{i} a_{i} \in \mathbb{Z}
$$

Each individual $a_{i}$ was previously only known to be a rational number [Yos14, Th. 0.1]. In fact, Yoshikawa has obtained an explicit expression for $a_{i}$, but it is too complicated. Nevertheless, if the singular fibers of $f$ has at most ODP singularities, then $a_{i}$ has a simple form. Combining this with Theorem 0.1, we get

Corollary 0.3. If the singular fibers of $f$ has at most ODP singularities, then

$$
(\chi+36) \operatorname{deg} \mathcal{H}_{e}^{3,0}+12 \operatorname{deg} \mathcal{H}_{e}^{2,1}=2 \sum_{j} \Delta_{j},
$$

where $\Delta_{j}=\# \operatorname{Sing} X_{j}$ is the number of ODP's on $X_{j}$.

Note that (0.3) is a special case of (0.1). Conversely, by comparing (0.1) and (0.2), we have the following geometric meaning for $12 \sum_{i} a_{i}$ :

\footnotetext{
${ }^{1}$ In their paper, this inequality is strict which we believe need further explanation. Through email correspondence, Kerr has told us that he agrees with this point.
} 
Corollary 0.4. If $f$ is relatively minimal and has at most semi-stable singular fibers, then

$$
12 \sum_{i} a_{i}=\sum_{i}\left\{\chi_{2}^{i}-3 I_{2}^{i}\right\}
$$

By comparing the two sides of this equality, it is reasonable to expect the following

Conjecture 0.5. If $f$ is relatively minimal and the singular fiber $f^{-1}\left(s_{i}\right)$ is semistable, then

$$
12 a_{i}=\chi_{2}^{i}-3 I_{2}^{i}
$$

In this direction, Eriksson-Freixas-Mourougane [EFM16] has obtained interesting results for the asymptotic expansion of the BCOV metric. Recently, they informed us that they have solved this conjecture positively.

On the other hand, by using the curvature properties of the moduli space of polarized Calabi-Yau manifolds and Yau's Schwarz lemma, we prove the following Arakelov type inequalities:

Theorem 0.6. 1. for $n=3$, we have

(i) $f$ is isotrivial $\Leftrightarrow \operatorname{deg} \mathcal{H}_{e}^{3,0}=0 \Leftrightarrow 12 \sum_{i} a_{i}-\chi \operatorname{deg} \mathcal{H}_{e}^{3,0}=0$;

(ii) if $f$ is non-isotrivial, then

$$
0<2 \operatorname{deg} \mathcal{H}_{e}^{3,0} \leq \sum_{i} a_{i}-\frac{\chi}{12} \operatorname{deg} \mathcal{H}_{e}^{3,0} \leq 2 \pi(2 g-2+s)\left[\left(\sqrt{h^{2,1}}+1\right)^{2}+1\right] .
$$

2. for $n=4$, we have

(i) $f$ is isotrivial $\Leftrightarrow \operatorname{deg} \mathcal{H}_{e}^{4,0}=0 \Leftrightarrow 2 \operatorname{deg} \mathcal{H}_{e}^{4,0}+\operatorname{deg} \mathcal{H}_{e}^{3,1}=0$;

(ii) if $f$ is non-isotrivial, then

$$
0<\operatorname{deg} \mathcal{H}_{e}^{4,0} \leq 2 \operatorname{deg} \mathcal{H}_{e}^{4,0}+\operatorname{deg} \mathcal{H}_{e}^{3,1} \leq \pi(2 g-2+s)\left(h^{3,1}+4\right) .
$$

In Section VI of [GGK09], more refined Arakelov inequalities (not necessarily for Calabi-Yau manifolds) for $n=1,2,3$ is proved and their proof is Hodge-theoretic. It should be pointed out that our Schwarz lemma approach is also suited to proving general Arakelov inequality for (abstract) variation of Hodge structures by using the curvature computations in [GS69].

For $n=3$, we have the following bound on the Euler number $\chi$ of the general fiber of $f$ :

Corollary 0.7. If $f: \mathcal{X} \rightarrow S$ is not isotrivial, then

$$
\frac{\sum_{i} a_{i}-2 \pi(2 g-2+s)\left[\left(\sqrt{h^{2,1}}+1\right)^{2}+1\right]}{\operatorname{deg} \mathcal{H}_{e}^{3,0}} \leq \frac{\chi}{12} \leq \frac{\sum_{i} a_{i}}{\operatorname{deg} \mathcal{H}_{e}^{3,0}}-2 .
$$

Theorem 0.8. If $f: \mathcal{X} \rightarrow S$ has no singular fibers and is not isotrivial.

(i) for $n=3$, we have

$$
\frac{-\pi(2 g-2)\left[\left(\sqrt{h^{2,1}}+1\right)^{2}+1\right]}{\operatorname{deg} \mathcal{H}^{3,0}} \leq \frac{\chi}{24} \leq-1 ;
$$


(ii) for $n=4$, we have

$$
1-\frac{\operatorname{deg} \mathcal{H}^{2,1}}{2 \operatorname{deg} \mathcal{H}^{4,0}} \leq \frac{\chi}{24} \leq \frac{2 \pi(2 g-2)\left(h^{3,1}+4\right)-\operatorname{deg} \mathcal{H}^{2,1}}{2 \operatorname{deg} \mathcal{H}^{4,0}}
$$

In the $n=3$ case, the Euler number is given by $\chi=2\left(h^{1,1}-h^{2,1}\right)$, so we see that a non-isotrivial family of Calabi-Yau threefolds with $h^{2,1}=1$ (mirror quintic type) must have singular fibers, as was shown in [GGK09]. In the $n=4$ case, assume $\omega_{H^{3}}=c_{1}\left(\mathcal{H}^{2,1}\right)=0$, then it was shown in [FL05] that if $\chi>24\left(h^{3,1}+2\right)$ or $\chi<24$, there exists no complete curve in $\mathcal{M}$, the moduli space of polarized CalabiYau manifolds. In particular, if $f$ has no singular fibers, then $1 \leq \frac{\chi}{24} \leq h^{3,1}+2$. If, however, we assume the total space $\mathcal{X}$ has trivial canonical bundle, then it was shown in [TZ13] that $f$ must be isotrivial.

We would like to remark that the results in this note are consequences of known results. Theorem 0.1 can be viewed as an integrated version of a theorem of Fang, $\mathrm{Lu}$, Yoshikawa. Conjecture 0.5 is obtained by comparing Theorem 0.1 and a result of Green, Griffiths, Kerr. The characterization of isotriviality in Theorem 0.6 is an interpretation of the vanishing of the integrals of the Weil-Petersson form and the Hodge form. The Arakelov inequality in Theorem 0.6 is a consequence of the known curvature bound of the Hodge metric and Yau's Schwartz Lemma. The bound on the Euler number is a reformulation of the Arakelov inequality. What we do in this paper is to bring together the works of two different groups, Fang-Lu-Yoshikawa on the one hand and Green-Griffiths-Kerr on the other. While Fang-Lu-Yoshikawa concentrate in local problems, Green-Griffiths-Kerr is more interested in global results. By transforming from local to global, and from global to local, we build a connection between analytical method in Fang-Lu-Yoshikawa and algebraic method in GreenGriffiths-Kerr. We find that their works can in fact help each other and enable us to know more about the nature of BCOV invariants and degenerations of CalabiYau manifolds. We now know that the asymptotic values of BCOV invariants are essentially integers, in fact they reflect the topological and intersection data of singular fibers which is not clear in previous works. Also the Euler number bound provides partial evidence for a conjecture of Yau which states that there are at most finitely many topological types of Calabi-Yau manifolds in each dimension, compare [Hun90].

This paper is organized as follows. In Section 1, we introduce the notion of BCOV invariant; In Section 2, we recall the definitions and basic properties of the WeilPetersson metric, generalized Hodge metrics and Hodge bundles; In Section 3, we show that the total singularities can be expressed by the sum of asymptotic values of BCOV invariants. In Section 4, by using Yau's Schwarz lemma, we prove Arakelov type inequalities and Euler number bound for Calabi-Yau family over a compact Riemann surface.

Acknowledgements. The second author would like to thank Professors Carlos Simpson and Matt Kerr for useful discussions. We would like to thank the referees for carefully reading our manuscript and their valuable comments. We would also like to thank Eriksson, Freixas and Mourougane for their useful comments. The work of 
K. Liu is partially supported by the National Natural Science Foundation of China, Grant No. 11531012.

\section{BCOV invariants}

In this section, we introduce the notion of BCOV invariant. We will mainly follow the presentation in [Yos14], see [FLY08] for more details.

1.1. Analytic torsion and BCOV torsion. Let $(M, g)$ be a compact Kähler manifold of dimension $n$ with Kähler form $\omega$. Let $\square_{p, q}=\left(\bar{\partial}+\bar{\partial}^{*}\right)^{2}$ be the $\bar{\partial}$-Laplacian acting on $C^{\infty}(p, q)$-forms on $M$ or equivalently $(0, q)$-forms on $M$ with values in $\Omega_{M}^{p}$, where $\Omega_{M}^{1}$ is the holomorphic cotangent bundle of $M$ and $\Omega_{M}^{p}:=\Lambda^{p} \Omega_{M}^{1}$. Let $\left\{\lambda_{j}\right\}$ be the eigenvalues of $\square_{p, q}$, then it is well-known that $0=\lambda_{0} \leq \lambda_{1} \leq \lambda_{2} \leq \cdots \leq \lambda_{j} \longrightarrow+\infty$. The spectral zeta function of $\square_{p, q}$ is defined as

$$
\zeta_{p, q}(s):=\sum_{\lambda_{j}>0} \lambda_{j}^{-s}
$$

where the multiplicities of the eigenvalues are taken into account. Then $\zeta_{p, q}(s)$ converges on the half-plane $\{s \in \mathbb{C} ; \Re s>\operatorname{dim} M\}$, extends to a meromorphic function on $\mathbb{C}$, and is holomorphic at $s=0$. By Ray-Singer [RS73], the analytic torsion of $\left(M, \Omega_{M}^{p}\right)$ is the real number defined as

$$
\tau\left(M, \Omega_{M}^{p}\right):=\exp \left\{-\sum_{q \geq 0}(-1)^{q} q \zeta_{p, q}^{\prime}(0)\right\} .
$$

Note that $\tau\left(M, \Omega_{M}^{p}\right)$ depends not only on the complex structure of $M$ but also on the metric $g$.

In [BCOV94], Bershadsky-Cecotti-Ooguri-Vafa introduced the following combination of analytic torsions.

Definition 1.1. The BCOV torsion of $(M, g)$ is the real number defined as

$$
T_{\mathrm{BCOV}}(M, g):=\prod_{q \geq 0} \tau\left(M, \Omega_{M}^{p}\right)^{(-1)^{p}}=\exp \left\{-\sum_{p, q \geq 0}(-1)^{p+q} p q \zeta_{p, q}^{\prime}(0)\right\} .
$$

If $\omega$ is the Kähler form of $g$, then we often write $T_{\mathrm{BCOV}}(M, \omega)$ for $T_{\mathrm{BCOV}}(M, g)$. In general, $T_{\mathrm{BCOV}}(M, g)$ does depend on the choice of Kähler metric $g$ and hence is not a holomorphic invariant of $M$. When $M$ is a Calabi-Yau threefold, it is possible to construct a holomorphic invariant of $M$ from $T_{\mathrm{BCOV}}(M, g)$ by multiplying a correction factor.

1.2. Calabi-Yau threefolds and BCOV invariants. A compact connected Kähler manifold $X$ is Calabi-Yau if $H^{q}\left(X, \mathcal{O}_{X}\right)=0$ for $0<q<\operatorname{dim} X$ and $K_{X} \cong \mathcal{O}_{X}$, where $K_{X}$ is the canonical line bundle of $X$. Let $X$ be a Calabi-Yau threefold. Let $g=\sum_{i, j} g_{i \bar{j}} d z_{i} \otimes d \bar{z}_{j}$ be a Kähler metric on $X$ and let $\omega=\omega_{g}:=\sqrt{-1} \sum_{i, j} g_{i \bar{j}} d z_{i} \wedge d \bar{z}_{j}$ be the corresponding Kähler form. we define

$$
\operatorname{Vol}(X, \omega):=\frac{1}{(2 \pi)^{3}} \int_{X} \frac{\omega^{3}}{3 !} .
$$


The covolume of $H^{2}(X, \mathbb{Z})_{\text {free }}:=H^{2}(X, \mathbb{Z}) /$ Torsion with respect to $[\omega]$ is defined as

$$
\operatorname{Vol}_{L^{2}}\left(H^{2}(X, \mathbb{Z}),[\omega]\right):=\operatorname{det}\left(\left\langle e_{i}, e_{j}\right\rangle_{L^{2},[\omega]}\right)_{1 \leq i, j \leq b_{2}(X)} .
$$

Here $\left\{e_{1}, \ldots, e_{b_{2}(X)}\right\}$ is a basis of $H^{2}(X, \mathbb{Z})_{\text {free }}=\operatorname{Im}\left\{H^{2}(X, \mathbb{Z}) \rightarrow H^{2}(X, \mathbb{R})\right\}$ over $\mathbb{R}$ and $\langle\cdot, \cdot\rangle_{L^{2},[\omega]}$ is the inner product on $H^{2}(X, \mathbb{R})$ induced by integration of harmonic forms.

Definition 1.2. For a Calabi-Yau threefold $X$ equipped with a Kähler form, define

$$
A(X, \omega):=\exp \left[-\frac{1}{12} \int_{X} \log \left(\sqrt{-1} \frac{\eta \wedge \bar{\eta}}{\omega^{3} / 3 !} \frac{\operatorname{Vol}(X, \omega)}{\|\eta\|_{L^{2}}^{2}}\right) c_{3}(X, \omega)\right],
$$

where $c_{3}(X, \omega)$ is the top Chern form of $(X, \omega), \eta \in H^{0}\left(X, K_{X}\right)$ is a nowhere vanishing section and $\|\eta\|_{L^{2}}$ is its $L^{2}$-norm, i.e.,

$$
\|\eta\|_{L^{2}}:=\frac{1}{(2 \pi)^{3}} \int_{X} \sqrt{-1} \eta \wedge \bar{\eta}
$$

Note that $A(X, \omega)$ is independent of the choice of $\eta$ and $A(X, \omega)=1$ if $\omega$ is Ricciflat.

Definition 1.3. The BCOV invariant of $X$ is the real number defined as

$$
\tau_{\mathrm{BCOV}}(X):=\operatorname{Vol}(X, \omega)^{-3+\frac{\chi(X)}{12}} \operatorname{Vol}_{L^{2}}\left(H^{2}(X, \mathbf{Z}),[\omega]\right)^{-1} T_{\mathrm{BCOV}}(X, \omega) A(X, \omega) .
$$

It turns out $\tau_{\mathrm{BCOV}}(X)$ is independent of the metric chosen.

Theorem 1.4. [FLY08, Th. 4.16] For a Calabi-Yau threefold $X, \tau_{\mathrm{BCOV}}(X)$ is independent of the choice of a Kähler form on $X$.

So we see that if $\pi: \mathcal{X} \rightarrow S$ is a smooth family of Calabi-Yau threefolds, then $\tau_{\mathrm{BCOV}}(t):=\tau_{\mathrm{BCOV}}\left(X_{t}\right), \forall t \in S$, defines a smooth function on $\mathrm{S}$, where the smoothness is proved in [BGS88c, Cor. 3.9].

2. Weil-Petersson metric, generalized Hodge metrics and Hodge bundles

Let $(X,[\omega])$ be a polarized Calabi-Yau manifold of dimension $n \geq 3$, that is, $X$ is a Calabi-Yau manifold and $[\omega]=c_{1}(L) \in H^{2}(X, \mathbb{Z})$ is the first Chern class of a positive line bundle $L$ on $X$. Let $\mathcal{M}$ be the (coase) moduli space of the polarized Calabi-Yau manifold $(X,[\omega])$. By Viehweg [Vie95], $\mathcal{M}$ is quasi-projective. Locally, $\mathcal{M}$ is identified as a finite discrete quotient of the local versal deformation space Def (Kuranishi space) of $X$. By the Bogomolov-Tian-Todorov theorem [Tia87, Tod89], the base space (which is a priori only a complex analytic space) Def of the Kuranishi family

$$
\pi:(\mathfrak{X}, X) \rightarrow(\text { Def }, 0)
$$

is smooth. Indeed, Def is an open subset of the linear space $H^{1}\left(X, \Theta_{X}\right)$, where $\Theta_{X}$ is the holomorphic tangent bundle of $X$, so we may assume Def is contractible. Since $H^{0}\left(X, \Theta_{X}\right) \cong H^{0}\left(X, \Omega_{X}^{n-1}\right)=0$, the Kuranishi family $\pi$ is universal. Define

$$
\left.H^{1}\left(X, \Theta_{X}\right)_{\omega}:=\left\{\theta \in H^{1}\left(X, \Theta_{X}\right) \mid \theta\right\lrcorner \omega=0 \in H^{2}\left(X, \mathcal{O}_{X}\right)\right\}
$$


and $\operatorname{Def}_{\omega}:=\operatorname{Def} \cap \mathrm{H}^{1}\left(\mathrm{X}, \Theta_{\mathrm{X}}\right)_{\omega}$, then Def $\operatorname{Den}_{\omega}$ consists of those local deformations of $X$ preserving the polarization $[\omega]$. In fact, since $H^{2}\left(X, \mathcal{O}_{X}\right)=0$, we have Def $f_{\omega}=\operatorname{Def}$ We thus have the associated period mapping Def $\rightarrow \mathrm{D}$, where $D$ is the period domain, i.e. the classifying space of polarized (weight $k$ ) Hodge structure of $(X,[\omega])$. By Griffiths [Gri68, Cor.3.6], Def $\rightarrow$ D is a holomorphic mapping, and is an immersion (Local Torelli) for $k=n$. We remark that $\mathcal{M}$ is an orbifold and is locally covered by Def, so when we work with metrics, curvatures of $\mathcal{M}$, we can treat these notions on Def instead.

Now we recall some natural metrics on $\mathcal{M}$, see [FL05] for more details.

2.1. Weil-Petersson metric. Let $t \in$ Def, the Kodaira-Spencer map is now an isomorphism

$$
\rho: T_{t} \text { Def } \stackrel{\cong}{\rightarrow} \mathrm{H}^{1}\left(\mathrm{X}_{\mathrm{t}}, \Theta_{\mathrm{t}}\right),
$$

where $\Theta_{t}$ is the holomorphic tangent bundle of $X_{t}$.

Let $\left(t_{1}, \cdots, t_{m}\right)$ be a local holomorphic coordinate system of $\mathcal{M}$, we define a Hermitian inner product on $T_{t} \mathcal{M}$ by

$$
\left(\frac{\partial}{\partial t_{i}}, \frac{\partial}{\partial \bar{t}_{j}}\right)_{W P}=\int_{X_{t}} A_{i \bar{\beta}}^{\alpha} \cdot \overline{A_{j \bar{\delta}}^{\gamma}} g^{\delta \bar{\beta}} g_{\alpha \bar{\gamma}} d V_{X_{t}}
$$

where $A_{i}=A_{i \bar{\beta}}^{\alpha} \frac{\partial}{\partial t_{\alpha}} \otimes d \bar{t}^{\beta},(i=1, \cdots, m)$ are the harmonic representation of $\rho\left(\frac{\partial}{\partial t_{i}}\right)$. This inner product on each $T_{t} U$ for $t \in \mathcal{M}$ gives a Hermitian metric on the moduli space $\mathcal{M}$, which is called the Weil-Petersson metric. Equipped with the WeilPetersson metric, $\mathcal{M}$ is a Kähler orbifold.

Let $\Omega$ be a (nonzero) holomorphic $(n, 0)$-form on $X_{t}$. Define $\left.\Omega\right\lrcorner \rho\left(\frac{\partial}{\partial t_{i}}\right)$ to be the contraction of $\Omega$ and $\rho\left(\frac{\partial}{\partial t_{i}}\right)$. The Weil-Petersson metric can be re-written as (cf. [Tia87]):

$$
\left(\frac{\partial}{\partial t_{i}}, \frac{\partial}{\partial \bar{t}_{j}}\right)_{W P}=-\frac{\left.\int_{X_{t}} \Omega\right\lrcorner \rho\left(\frac{\partial}{\partial t_{i}}\right) \wedge \overline{\Omega\lrcorner \rho\left(\frac{\partial}{\partial t_{j}}\right)}}{\int_{X_{t}} \Omega \wedge \bar{\Omega}} .
$$

2.2. Generalized Hodge metrics and Hodge bundles. Recall that, for all $0 \leq$ $k \leq n$, and $p+q=k$ there are natural holomorphic vector bundles $P R^{q} \pi_{*} \Omega_{\mathfrak{X} / \text { Def }}^{p}$, called Hodge bundles, on Def (hence on $\mathcal{M}$ ), whose fiber is

$$
\left(P R^{q} \pi_{*} \Omega_{\mathfrak{X} / \text { Def }}^{p}\right)_{t}=P H^{q}\left(X_{t}, \Omega_{X_{t}}^{p}\right),
$$

where $P H^{q}\left(X_{t}, \Omega_{X_{t}}^{p}\right)$ is the primitive cohomology of $X_{t}$. By abuse of notation ,we will always use the same symbol $\mathcal{H}^{p, q}$ to denote the Hodge bundle on Def and on $\mathcal{M}$.

By differentiating harmonic representatives, we have a holomorphic bundle map

$$
\frac{\partial}{\partial t_{i}}: \mathcal{F}^{p} \rightarrow \mathcal{H} / \mathcal{F}^{p}
$$

where $\mathcal{F}^{p}=\mathcal{H}^{p, k-p} \oplus \mathcal{H}^{p+1, k-p-1} \oplus \cdots \oplus \mathcal{H}^{k, 0}, \mathcal{H}:=P R^{k} \pi_{*}(\mathbb{C})$. In this way, we get a natural holomorphic bundle map

$$
T(\text { Def }) \rightarrow \underset{1 \leq \mathrm{p} \leq \mathrm{k}}{\oplus} \operatorname{Hom}\left(\mathcal{F}^{\mathrm{p}}, \mathcal{H} / \mathcal{F}^{\mathrm{p}}\right)
$$


Note that, this bundle map is just the differential of the period mapping Def $\rightarrow \mathrm{D}$. There are natural metrics on the Hodge bundles $\mathcal{F}^{p}$, hence on each $\operatorname{Hom}\left(\mathcal{F}^{p}, \mathcal{H} / \mathcal{F}^{p}\right)$, induced by the Riemann-Hodge bilinear relations. Let $h_{P H^{k}}$ be the pull back of the metric on $\underset{1 \leq p \leq k}{\oplus} \operatorname{Hom}\left(\mathcal{F}^{p}, \mathcal{H} / \mathcal{F}^{p}\right)$ by $(2.5)$, then $h_{P H^{k}}$ is semi-positive. We use $\omega_{P H^{k}}$ to denote the Kähler form of $h_{P H^{k}}$ for all $k \leq n$. And let

$$
\omega_{H^{k}}:=\omega_{P H^{k}}+\omega_{P H^{k-2}}+\cdots .
$$

We call both $\omega_{H^{k}}$ and $\omega_{P H^{k}}$ to be the generalized Hodge metrics. We note that when $k=n, \omega_{P H^{n}}$ is a positive $(1,1)$ form by local Torelli. It's just the pull back of the usual Hodge metric on $D$ and we will call $\omega_{H}:=\omega_{P H^{n}}$ the Hodge metric. There are several interesting relations between these metrics and Hodge bundles:

Lemma 2.1. Let $m=\operatorname{dim} \mathcal{M}=h^{n-1,1}$, then we have

(1) for $n=3, \omega_{H}=(m+3) \omega_{W P}+\operatorname{Ric}\left(\omega_{W P}\right)$;

(2) for $n=4, \omega_{H}=(2 m+4) \omega_{W P}+2 \operatorname{Ric}\left(\omega_{W P}\right)$;

(3) $\omega_{P H^{k}}=\sum_{0 \leq p \leq k} p c_{1}\left(\mathcal{H}^{p, q}\right)$;

(4) $\omega_{H^{k}}=\sum_{0 \leq p \leq k} p c_{1}\left(R^{k-p} \pi_{*} \Omega_{\mathfrak{X} / \mathrm{Def}}^{p}\right)$;

(5) $\omega_{W P}=c_{1}\left(\mathcal{H}^{n, 0}\right)$.

Proof. (1) is in [Lu01, Thm. 1.2], see also [Wan03, Thm.3.2] for an alternative proof; (2) is in [LS04, Coro. 6.4]; (3),(4) is in [FL05, Prop. 2.8]; (5) is in [Tia87, Thm. 2].

Let us consider the Hodge metric $\omega_{H}$ on $\mathcal{M}$ which is the pull-back of the homogeneous metric on the period domain $D$ by the period map. For Calabi-Yau threefolds, it can also be defined by (1) of Lemma 2.1, and is shown, up to a constant, to be the pull back of the usual Hodge metric on period domain $D$. Hence these are equivalent definitions. The holomorphic sectional curvature of $\mathcal{M}$ under Weil-Petersson metric is not negative in general. The Hodge metric has better curvature property, indeed, the holomorphic sectional curvature is bounded from above by $\frac{-1}{(\sqrt{m}+1)^{2}+1}$ for Calabi-Yau threefolds. For Calabi-Yau fourfolds, the upper bound is $\frac{-1}{m+4}$. See [Lu01, LS04].

2.3. Weil-Petersson form and Hodge form. Let $f: \mathcal{X} \rightarrow S$ be a smooth polarized family of Calabi-Yau manifolds with $\left(X_{0}:=f^{-1}(0),\left[\omega_{0}\right]\right) \cong(X,[\omega])$, where $0 \in S$ and $\left[\omega_{0}\right]$ is the polarization of $X_{0}$ induced from that of $\mathcal{X}$. Since $\mathcal{M}$ is the moduli space of the polarized Calabi-Yau manifold $(X,[\omega])$, we have a natural commutative diagram :

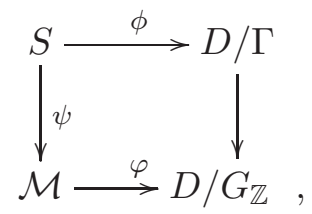

where $\Gamma$ is the monodromy group of the family $f$, and $G_{\mathbb{Z}}=A u t\left(H_{\mathbb{Z}}, Q\right)$, see [Gri84] for more information. To make notations simple, we will just use $\omega_{W P}$ and $\omega_{H}$ to denote the pull back form $\psi^{*} \omega_{W P}$ and $\psi^{*} \omega_{H}$, and call them the Weil-Petersson form and Hodge form on $S$, respectively. Similarly, we will use $\mathcal{H}^{p, q}$ to denote the pull back 
bundle $\psi^{*} \mathcal{H}^{p, q}$, which is isomorphic to $P R^{q} f_{*} \Omega_{\mathcal{X} / S}^{p}$, and call them Hodge bundles on $S$.

3. Total singularities in terms of asymptotic values of BCOV invariants

Let $\mathcal{X}$ be a smooth projective variety of dimension $n+1, S$ be a compact Riemann surface and $f: \mathcal{X} \rightarrow S$ be a surjective, flat holomorphic map with generic fiber CalabiYau $n$-fold. Let $f^{0}: \mathcal{X}^{0} \rightarrow S^{0}$ be the smooth part of $f$, that is, each fiber $f^{-1}(s)$ is smooth for $s \in S^{0}$ and singular for $s \notin S^{0}, \mathcal{X}^{0}=f^{-1}\left(S^{0}\right)$ and $f^{0}$ is the restriction of $f$ to $\mathcal{X}^{0}$. We will use these notations throughout this paper unless otherwise stated.

Now, assume $n=3$, let $0 \in S$ and $X_{0}=f^{-1}(0)$ be a singular fiber, $(U, t)$ be a coordinate neighborhood of $S$ centered at 0 . The following result is essential for us:

Theorem 3.1. [FLY08, Th. 10.1] Set

$$
a_{0}:=\lim _{t \rightarrow 0} \frac{\left.\log \tau_{\mathrm{BCOV}}\right|_{U}(t)}{\log |t|^{2}} \in \mathbb{R} .
$$

Then the following equation of currents on $U$ holds:

$$
d d^{c} \log \tau_{\mathrm{BCOV}}=-\frac{\chi}{12} \Omega_{\mathrm{WP}}-\Omega_{\mathrm{H}}+a_{0} \delta_{0},
$$

where $\tau_{\mathrm{BCOV}}(t):=\tau_{\mathrm{BCOV}}\left(X_{t}\right)$, for all $t \in S^{0}$, and $\log \tau_{\mathrm{BCOV}}$ is a locally integrable function on $S$. The currents $\Omega_{\mathrm{WP}}$ and $\Omega_{\mathrm{H}}$ are the trivial extensions of Weil-Petersson form and Hodge form from $S^{0}$ to $S$. Here $\delta_{0}$ is the Dirac current of the point 0 and $\chi$ is the topological Euler number of a general fiber of $f$.

Remark 3.2. In [FLY08, Prop. 7.3], it is proved that the Weil-Petersson form $\omega_{\mathrm{WP}}$ and Hodge form $\omega_{\mathrm{H}}$ are bounded by the Poincaré metric on the punctured unit disc $\Delta^{*}$, this implies that $\omega_{\mathrm{WP}}$ and $\omega_{\mathrm{H}}$ are locally integrable on $S$ and extend trivially to closed positive (1,1)-currents on $S$. Recall that the trivial extension is defined as follows: if $\tilde{T}$ is the trivial extension of the $(1,1)$-form $T$ from $\Delta^{*}$ to the unit disc $\Delta$,

$$
\tilde{T}(\eta):=\int_{\Delta} \eta T, \quad \eta \in C_{0}^{\infty}(\Delta) .
$$

Recall that an n-dimensional singularity is an ordinary double point (ODP for short) if it is isomorphic to the hypersurface singularity at $0 \in \mathbb{C}^{n+1}$ defined by the equation $z_{0}^{2}+z_{1}^{2}+\cdots+z_{n}^{2}=0$. And a fiber of $f: \mathcal{X} \rightarrow S$ is semi-stable if it is a reduced normal crossing divisor of $\mathcal{X}$.

If $X_{0}$ only has ODP's as singularities, then it was shown by Yoshikawa [Yos14, Th. 5.2], that $a_{0}=\frac{\# \operatorname{Sing} X_{0}}{6}$. Indeed,

$$
\log \tau_{\mathrm{BCOV}}\left(X_{t}\right)=\frac{\# \operatorname{Sing} X_{0}}{6} \log |t|^{2}+O(\log (-\log |t|)), \quad t \rightarrow 0 .
$$

The value of $a_{0}$ for semi-stable degenerations is also obtained in [Yos14, Th.3.11], but has a very complicated expression. Inspired by the work of Green-GriffithsKerr [GGK09], we conjecture that it should be expressed as the topological and intersecting data of the singular fiber, see Conjecture 3.9. 
Our first observation is that the Weil-Petersson form and Hodge form and their trivial current extensions are naturally related to Hodge bundles. To begin with, let $\omega_{W P}, \omega_{H}$ be the Weil-Petersson form and Hodge form on $S^{0}$, respectively. $\Omega_{\mathrm{WP}}$ and $\Omega_{\mathrm{H}}$ are their trivial extensions on $S$ defined by (3.2). On $S^{0}$, from Lemma 2.1 we know that

$$
\omega_{W P}=c_{1}\left(\mathcal{H}^{3,0}\right)
$$

and

$$
\omega_{H}=\omega_{P H^{3}}=\sum_{p=0}^{3} p c_{1}\left(\mathcal{H}^{p, 3-p}\right)=3 c_{1}\left(\mathcal{H}^{3,0}\right)+c_{1}\left(\mathcal{H}^{2,1}\right) .
$$

On the other hand, since we have assumed that all the local monodromy transformations around points $s_{i} \in S \backslash S^{0}$ are unipotent, there is then the canonical Deligne's extension $\mathcal{H}_{e}^{p, q}$ of the Hodge bundle $\mathcal{H}^{p, q}$ from $S^{0}$ to $S$. As is already remarked in [GGK09, p.475], it follows from Schmid's work [Sch73] that

(i) the forms $c_{1}\left(\mathcal{H}^{p, q}\right)$ are integrable and define closed, $(1,1)$ currents $\overline{c_{1}\left(\mathcal{H}^{p, q}\right)}$ (trivial extensions) on the completion $S$;

(ii) $\overline{c_{1}\left(\mathcal{H}^{p, q}\right)}=c_{1}\left(\mathcal{H}_{e}^{p, q}\right)$ in $H_{D R}^{2}(S)$.

For a precise statement with a proof of (i) and (ii), we refer the reader to [CKS86, Coro. 5.23].

We make the following observations:

Lemma 3.3. As cohomology classes in $H_{D R}^{2}(S)$, we have

$$
\Omega_{\mathrm{WP}}=\overline{c_{1}\left(\mathcal{H}^{3,0}\right)}=c_{1}\left(\mathcal{H}_{e}^{3,0}\right),
$$

and

$$
\Omega_{\mathrm{H}}=3 \overline{c_{1}\left(\mathcal{H}^{3,0}\right)}+\overline{c_{1}\left(\mathcal{H}^{2,1}\right)}=3 c_{1}\left(\mathcal{H}_{e}^{3,0}\right)+c_{1}\left(\mathcal{H}_{e}^{2,1}\right) .
$$

Proof. This follows from (3.4),(3.5) and (ii).

We now have the following general picture:

Theorem 3.4 (=Theorem 0.1). Let $E:=S \backslash S^{0}=\left\{s_{i}\right\} \subset S$ be the discriminant locus of $f$ and $\tau_{\mathrm{BCOV}}(t)$ be the BCOV invariant of the fiber $f^{-1}(t)$. Set

$$
a_{i}:=\lim _{t \rightarrow s_{i}} \frac{\log \tau_{\mathrm{BCOV}}(t)}{\log |t|^{2}} .
$$

Then

$$
\text { Total Singularities }=(\chi+36) \operatorname{deg} \mathcal{H}_{e}^{3,0}+12 \operatorname{deg} \mathcal{H}_{e}^{2,1}=12 \sum_{i} a_{i} .
$$

Proof. First note that by Theorem 3.1, we have on $S$ the following current equation

$$
d d^{c} \log \tau_{\mathrm{BCOV}}=-\frac{\chi(X)}{12} \Omega_{\mathrm{WP}}-\Omega_{\mathrm{H}}+\sum_{i} a_{i} \delta_{s_{i}} .
$$


By (3.6), (3.7), we then have the following equality of cohomology classes in $H_{D R}^{2}(S)$ :

$$
d d^{c} \log \tau_{\mathrm{BCOV}}=-\frac{\chi+36}{12} c_{1}\left(\mathcal{H}_{e}^{3,0}\right)-c_{1}\left(\mathcal{H}_{e}^{2,1}\right)+\sum_{i} a_{i} \delta_{s_{i}} .
$$

By integration on $S$, and noting that $d d^{c} \log \tau_{\mathrm{BCOV}}$ is an exact current on $S$, we get

$$
(\chi+36) \operatorname{deg} \mathcal{H}_{e}^{3,0}+12 \operatorname{deg} \mathcal{H}_{e}^{2,1}=12 \sum_{i} a_{i}
$$

It was shown in [Yos14, Th. 3.11], that for each $i, a_{i} \in \mathbb{Q}$. Now from (3.8), we see that

\section{Corollary 3.5.}

$$
12 \sum_{i} a_{i} \in \mathbb{Z}
$$

Remark 3.6. By the monodromy theorem (see, e.g., [Gri84, p.41]), the local monodromy transformations $T_{i}$ around points $s_{i}$ in the discriminant locus $E=S \backslash S^{0}$ are quasi-unipotent, and already unipotent for semi-stable degenerations (see [KK98, p.185], [Lan73]). In the general quasi-unipotent case, we may pass to the unipotent case by a sequence of base changes, or even better, to the semi-stable case by a sequence of semi-stable reductions, then if $\tilde{a}_{i}$ is the the asymptotic value for the semi-stable family, the asymptotic value for the original family is $a_{i}=\frac{\tilde{a_{i}}}{d_{i}}$, where $d_{i}$ is the degree of the base change corresponding to a neighborhood of $s_{i}$. See [Yos14, Th. 3.12]. And (3.8) becomes

$$
(\chi+36) \operatorname{deg} \mathcal{H}_{e}^{3,0}+12 \operatorname{deg} \mathcal{H}_{e}^{2,1}=12 \sum_{i} \tilde{a}_{i}=12 \sum_{i} a_{i} d_{i},
$$

where $\mathcal{H}_{e}^{3,0}$ and $\mathcal{H}_{e}^{2,1}$ are bundles on the base curve of the semi-stable family.

Now, assume $f$ has no singular fibers other than those with ODP's, denote these fibers by $X_{j}=f^{-1}\left(s_{j}\right), s_{j} \in E$. And let $\Delta_{j}=\# \operatorname{Sing} X_{j}$ be the number of ODP's on $X_{j}$. By Theorem 3.4 and (3.3), we immediately get

Corollary 3.7. If the singular fibers of $f$ has at most ODP singularities, then

$$
(\chi+36) \operatorname{deg} \mathcal{H}_{e}^{3,0}+12 \operatorname{deg} \mathcal{H}_{e}^{2,1}=12 \sum_{j} a_{j}=2 \sum_{j} \Delta_{j} .
$$

Furthermore, if we allow $f$ to have semi-stable fibers (i.e. reduced normal crossing divisors), and assume that $f$ is relatively minimal, then one of the main results in [GGK09, Th. (V.A.1)(b)] says the following:

$$
(\chi+36) \operatorname{deg} \mathcal{H}_{e}^{3,0}+12 \operatorname{deg} \mathcal{H}_{e}^{2,1}=2 \sum_{j} \Delta_{j}+\sum_{i}\left\{\chi_{2}^{i}-3 I_{2}^{i}\right\},
$$


where the second sum is over all semi-stable fibers $\left\{X_{s_{i}}\right\}$, and if $X_{s_{i}}=\bigcup_{\alpha} X_{\alpha}^{i}, X_{\alpha \beta}^{i}=$ $X_{\alpha}^{i} \cap X_{\beta}^{i}$, then

$$
\begin{gathered}
\chi_{2}^{i}:=\sum_{\alpha<\beta} \chi\left(X_{\alpha \beta}^{i}\right), \\
I_{2}^{i}:=\sum_{\alpha<\beta} \operatorname{deg}\left[\left(X_{\alpha \beta}^{i}\right)^{2}\right]-\operatorname{deg}\left[\left(\sum_{\alpha<\beta} X_{\alpha \beta}^{i}\right)^{2}\right] .
\end{gathered}
$$

So we see that (3.10) is a special case of (3.11) when there are only ODP singular fibers. This shouldn't be too surprising because our approach relies essentially on the curvature formula of determinant bundle [BGS88a, Th. 0.1], which can be viewed as a differential form version of the Grothendieck-Riemann-Roch formula (GRR for short).

As another example, we consider a family $f: X \rightarrow B$ of curves of genus $g$ over a compact Riemann surface $B$ of genus $q$, assume the fibers of $f$ have at most ODP singularities, then a direct application of the GRR formula will give us:

$$
12 \operatorname{deg} f_{*} \omega_{X / B}=\omega_{X / B}^{2}+e(X)-4(g-1)(q-1),
$$

where $e(X)$ is the topological Euler number of the algebraic surface $X$. Since $e(X)=$ $4(g-1)(q-1)+\delta$, where $\delta$ is the sum of number of ODP's on each singular fiber, we then have

$$
12 \operatorname{deg} f_{*} \omega_{X / B}=\omega_{X / B}^{2}+\delta
$$

This formula and its variant are well-known to experts and can be deduced from the classical Riemann-Roch and Leray spectral sequence. The point is that it can be shown using analytic torsions. This is another manifestation of the equivalence of these two approaches we described above. In fact, let $\xi$ be a holomorphic vector bundle over $X, \lambda(\xi)$ be the determinant line bundle with fiber over $b \in B$ being $\left[\operatorname{det} H^{0}\left(X_{b}, \xi\right)\right]^{-1} \otimes \operatorname{det} H^{1}\left(X_{b}, \xi\right)$, in other words, $\lambda(\xi)=\left[\operatorname{det} f_{*} \xi\right]^{-1} \otimes \operatorname{det} R^{1} f_{*} \xi$, then a theorem [BB90, Th. 2.1] of Bismut and Bost says that

$$
c_{1}\left(\lambda(\xi),\|\|_{Q}\right)=-\int_{f}\left[T d\left(\omega_{X / B}^{-1}\right) \operatorname{ch}(\xi)\right]^{(4)}-\frac{1}{12}(r k \xi) \sum_{i} \Delta_{i} \delta_{s_{i}},
$$

where $\int_{f}$ means integration along a general fiber, [] ${ }^{(4)}$ denotes the degree 4 component, $\Delta_{i}=\# \operatorname{Sing} X_{s_{i}}$ is the number of ODP's on $X_{s_{i}}$ and $\delta_{s_{i}}$ is the Dirac current of the point $s_{i}$ as usual. Now, let $\xi=\mathcal{O}_{X}$, then

$$
c_{1}\left(\operatorname{det} R^{1} f_{*} \mathcal{O}_{X}\right)=-\frac{1}{12} c_{1}^{2}\left(\omega_{X / B}\right)-\frac{1}{12} \sum_{i} \Delta_{i} \delta_{s_{i}} .
$$

Note that $R^{1} f_{*} \mathcal{O}_{X}=\left(f_{*} \omega_{X / B}\right)^{\vee}$. The integration of (3.15) over the base curve $B$ will give us exactly (3.13).

Let us go back to the study of Calabi-Yau family, we have seen that the ODP case of (3.11) can be deduced from the current equation (3.1), conversely, we may use (3.11) to say something about the asymptotic values of the BCOV invariants in the semi-stable case, firstly we have: 
If $f$ is relatively minimal and has no singular fibers other than ODP's and semistable ones, then

$$
12\left(\sum_{j} a_{j}+\sum_{i} a_{i}\right)=2 \sum_{j} \Delta_{j}+\sum_{i}\left\{\chi_{2}^{i}-3 I_{2}^{i}\right\},
$$

where we have used different indices $i, j$ to indicate different type of singular fibers.

In particular, we have the following

Corollary 3.8. If $f$ is relatively minimal and has at most semi-stable singular fibers, then

$$
12 \sum_{i} a_{i}=\sum_{i}\left\{\chi_{2}^{i}-3 I_{2}^{i}\right\}
$$

Note that in the equality (3.17), for each fixed $i, a_{i}$ is determined by the local data of $f$ near the point $s_{i}$, and $\chi_{2}^{i}-3 I_{2}^{i}$ is determined by the fiber $X_{s_{i}}=f^{-1}\left(s_{i}\right)$, so it is reasonable to expect the following

Conjecture 3.9 (=Conjecture 0.5). If $f$ is relatively minimal and the singular fiber $f^{-1}\left(s_{i}\right)$ is semi-stable, then

$$
12 a_{i}=\chi_{2}^{i}-3 I_{2}^{i}
$$

Conversely, if we can prove conjecture 3.9, then we can recover (3.11).

4. Arakelov inequality and Euler number bound for Calabi-Yau manifolds

As in Section 3, let $\mathcal{X}$ be a smooth projective variety of dimension $n+1, S$ be a compact Riemann surface and $f: \mathcal{X} \rightarrow S$ be a surjective, flat holomorphic map with generic fiber Calabi-Yau $n$-fold. The smooth part $f^{0}: \mathcal{X}^{0} \rightarrow S^{0}$ of $f$ is a polarized family of Calabi-Yau $n$-folds with the polarization induced from that of $\mathcal{X}$. Remember that we always assume that all the local monodromy transformations around points $s_{i} \in S \backslash S^{0}$ are unipotent. Following ideas in [Liu96b, Liu96a], we prove Arakelov type inequalities by using curvature properties of the moduli space of Calabi-Yau manifolds and Yau's Schwarz lemma. As is explained in [Liu96a], Yau's Schwarz lemma [Yau78a, Th. 2'] is still applicable if the target manifold is an orbifold.

There are lots of work on Arakelov inequality in the literature, see [GGK09, Pet00, VZ05] and the references therein.

4.1. General Calabi-Yau family. Let $E=S \backslash S^{0}$ be the discriminant locus, $s:=$ $\# E$ be the number of singular fibers of $f$. We will use the fact: $\omega_{H} \geq 2 \omega_{W P}$, which follows from the curvature property of Hodge bundles, see e.g. [FL05, Coro. 2.10].

Theorem 4.1 (=Theorem 0.6). 1. for $n=3$, we have

(i) $f$ is isotrivial $\Leftrightarrow \operatorname{deg} \mathcal{H}_{e}^{3,0}=0 \Leftrightarrow 12 \sum_{i} a_{i}-\chi \operatorname{deg} \mathcal{H}_{e}^{3,0}=0$;

(ii) if $f$ is not isotrivial, then

$$
0<2 \operatorname{deg} \mathcal{H}_{e}^{3,0} \leq \sum_{i} a_{i}-\frac{\chi}{12} \operatorname{deg} \mathcal{H}_{e}^{3,0} \leq 2 \pi(2 g-2+s)\left[\left(\sqrt{h^{2,1}}+1\right)^{2}+1\right] .
$$

2. for $n=4$, we have

(i) $f$ is isotrivial $\Leftrightarrow \operatorname{deg} \mathcal{H}_{e}^{4,0}=0 \Leftrightarrow 2 \operatorname{deg} \mathcal{H}_{e}^{4,0}+\operatorname{deg} \mathcal{H}_{e}^{3,1}=0$; 
(ii) if $f$ is not isotrivial, then

$$
0<\operatorname{deg} \mathcal{H}_{e}^{4,0} \leq 2 \operatorname{deg} \mathcal{H}_{e}^{4,0}+\operatorname{deg} \mathcal{H}_{e}^{3,1} \leq \pi(2 g-2+s)\left(h^{3,1}+4\right) .
$$

Proof. 1.(i) First, $f^{0}: \mathcal{X}^{0} \rightarrow S^{0}$ induces a holomorphic map from $S^{0}$ to the moduli space $\mathcal{M}$ of the polarized Calabi-Yau threefold $\left(X_{0},[\omega]\right)$ :

$$
\psi: S^{0} \rightarrow \mathcal{M},
$$

where $0 \in S^{0}$. Note that the Hodge form $\psi^{*} \omega_{H}$ on $S^{0}$ is semi-positive and locally integrable on $S$ (see Remark 3.2), hence $\int_{S^{0}} \psi^{*} \omega_{H}=0$ iff $\psi$ is constant which is equivalent to $f$ being isotrivial. Similarly, $\int_{S^{0}} \psi^{*} \omega_{W P}=0$ iff $f$ is isotrivial. But the Hodge form $\psi^{*} \omega_{H}$ and the Weil-Petersson form $\omega_{W P}$ have trivial current extensions on $S$ and by Lemma 3.3 and Theorem 3.4, we have

$$
\begin{gathered}
\int_{S^{0}} \psi^{*} \omega_{H}=\int_{S} \Omega_{H}=3 \operatorname{deg} \mathcal{H}_{e}^{3,0}+\operatorname{deg} \mathcal{H}_{e}^{2,1}=\sum_{i} a_{i}-\frac{\chi}{12} \operatorname{deg} \mathcal{H}_{e}^{3,0} \\
\int_{S^{0}} \psi^{*} \omega_{W P}=\int_{S} \Omega_{W P}=\operatorname{deg} \mathcal{H}_{e}^{3,0}
\end{gathered}
$$

so (i) follows.

(ii) By the uniformization theorem and Gauss-Bonnet theorem for punctured Riemann surfaces, $S^{0}$ admits a complete metric $\omega$ with constant curvature $K_{1}=+1,0,-1$ when the Euler number $e\left(S^{0}\right)=2-2 g-s$ is positive, zero or negative, respectively. Recall that the holomorphic sectional curvature of $\mathcal{M}$ with respect to the Hodge metric $\omega_{H}$ is bounded from above by $K_{2}=\frac{-1}{\left(\sqrt{h^{2,1}}+1\right)^{2}+1}$, so by Yau's Schwarz lemma, if $\psi$ is nonconstant, then $K_{1}=-1$ and

$$
\psi^{*} \omega_{H} \leq \frac{K_{1}}{K_{2}} \omega=\left[\left(\sqrt{h^{2,1}}+1\right)^{2}+1\right] \omega,
$$

by integration on $S^{0}$, we get

$$
\int_{S^{0}} \psi^{*} \omega_{H} \leq \frac{K_{1}}{K_{2}} \int_{S^{0}} \omega=2 \pi(2 g-2+s)\left[\left(\sqrt{h^{2,1}}+1\right)^{2}+1\right] .
$$

Now substituting equalities in (i) into this inequality, and using

$$
\int_{S^{0}} \psi^{*} \omega_{H} \geq 2 \int_{S^{0}} \psi^{*} \omega_{W P}>0
$$

we get 1.(ii).

2. The proof is almost the same as 1 . and is omitted.

Remark 4.2. Note that for $n=3$,

$$
2 \operatorname{deg} \mathcal{H}_{e}^{3,0}=\sum_{i} a_{i}-\frac{\chi}{12} \operatorname{deg} \mathcal{H}_{e}^{3,0} \Leftrightarrow \operatorname{deg} \mathcal{H}_{e}^{3,0}+\operatorname{deg} \mathcal{H}_{e}^{2,1}=0 .
$$

Now for $n=3$, we can give a bound on the Euler number $\chi$ of the general fiber of $f:$ 
Corollary 4.3. If $f: \mathcal{X} \rightarrow S$ is not isotrivial, then

$$
\frac{\sum_{i} a_{i}-2 \pi(2 g-2+s)\left[\left(\sqrt{h^{2,1}}+1\right)^{2}+1\right]}{\operatorname{deg} \mathcal{H}_{e}^{3,0}} \leq \frac{\chi}{12} \leq \frac{\sum_{i} a_{i}}{\operatorname{deg} \mathcal{H}_{e}^{3,0}}-2 .
$$

4.2. Non-degenerate Calabi-Yau family. It is well-known that a non-isotrivial family of elliptic curves over a compact Riemann surface must have singular fibers. This phenomena does not hold for higher dimensional Calabi-Yau manifold, see [GGK09] for such examples. For non-degenerate Calabi-Yau family, we have the following bound for the Euler number $\chi$ :

Theorem 4.4 (=Theorem 0.8). If $f: \mathcal{X} \rightarrow S$ has no singular fibers and is not isotrivial.

(i) for $n=3$, we have

$$
\frac{-\pi(2 g-2)\left[\left(\sqrt{h^{2,1}}+1\right)^{2}+1\right]}{\operatorname{deg} \mathcal{H}^{3,0}} \leq \frac{\chi}{24} \leq-1 ;
$$

(ii) for $n=4$, we have

$$
1-\frac{\operatorname{deg} \mathcal{H}^{2,1}}{2 \operatorname{deg} \mathcal{H}^{4,0}} \leq \frac{\chi}{24} \leq \frac{2 \pi(2 g-2)\left(h^{3,1}+4\right)-\operatorname{deg} \mathcal{H}^{2,1}}{2 \operatorname{deg} \mathcal{H}^{4,0}} .
$$

Proof. (i) is a direct consequence of Theorem 4.3. For (ii), first note that $f: \mathcal{X} \rightarrow S$ is now a polarized family of Calabi-Yau fourfolds with the polarization induced from that of $\mathcal{X}$. And recall Fang-Lu's formula [FL05, Th. 1.1]:

$$
\sum_{i=1}^{i=n}(-1)^{i} \omega_{H^{i}}-\frac{\sqrt{-1}}{2} \partial \bar{\partial} \log T=\frac{\chi}{12} \omega_{W P},
$$

where $\omega_{H^{i}}$ is defined in Section 2.2, $T$ is the BCOV torsion with respect to the unique Ricci-flat metric [Yau78b]. By Lemma 2.1,

$$
\omega_{H^{4}}-\omega_{H^{3}}+\omega_{H^{2}}-\omega_{H^{1}}=4 c_{1}\left(\mathcal{H}^{4,0)}\right)+2 c_{1}\left(\mathcal{H}^{3,1}\right)-c_{1}\left(\mathcal{H}^{2,1}\right),
$$

so by integration on $S$, we have

$$
4 \operatorname{deg} \mathcal{H}^{4,0}+2 \operatorname{deg} \mathcal{H}^{3,1}-\operatorname{deg} \mathcal{H}^{2,1}=\frac{\chi}{12} \operatorname{deg} \mathcal{H}^{4,0} .
$$

(4.6) follows from this and (4.2).

\section{References}

[BB90] J.-M. Bismut and J.-B. Bost. Fibrés déterminants, métriques de Quillen et dégénérescence des courbes. Acta Mathematica, 165(1):1-103, 1990.

[BCOV93] M. Bershadsky, S. Cecotti, H. Ooguri, and C. Vafa. Holomorphic anomalies in topological field theories. Nuclear Physics B, 405(2-3):279-304, 1993.

[BCOV94] M. Bershadsky, S. Cecotti, H. Ooguri, and C. Vafa. Kodaira-Spencer theory of gravity and exact results for quantum string amplitudes. Communications in Mathematical Physics, 165(2):311-427, 1994.

[BGS88a] J.-M. Bismut, H. Gillet, and C. Soulé. Analytic torsion and holomorphic determinant bundles i. Bott-Chern forms and analytic torsion. Communications in Mathematical Physics, 115(1):49-78, 1988. 
[BGS88b] J.-M. Bismut, H. Gillet, and C. Soulé. Analytic torsion and holomorphic determinant bundles ii. Direct images and Bott-Chern forms. Communications in Mathematical Physics, 115(1):79-126, 1988.

[BGS88c] J.-M. Bismut, H. Gillet, and C. Soulé. Analytic torsion and holomorphic determinant bundles iii. Quillen metrics on holomorphic determinants. Communications in Mathematical Physics, 115(2):301-351, 1988.

[Che79] J. Cheeger. Analytic torsion and the heat equation. Annals of Mathematics, 109(2):1-21, 1979 .

[CKS86] E. Cattani, A. Kaplan, and W. Schmid. Degeneration of Hodge structures. Annals of Mathematics, 123(3):457-535, 1986.

[EFM16] D. Eriksson, G. Freixas, and C. Mourougane. Singularities of metrics on Hodge bundles and their topological invariants. arXiv:math/1611.03017v1, 2016. To appear in Journal of Algebraic Geometry.

[FL05] H. Fang and Z. Lu. Generalized Hodge metrics and BCOV torsion on Calabi-Yau moduli. Journal für die reine und angewandte Mathematik, 588:49-69, 2005.

[FLY08] H. Fang, Z. Lu, and K.-I. Yoshikawa. Analytic torsion for Calabi-Yau threefolds. Journal of Differential Geometry, 80(2):175-259, 2008.

[GGK09] M. Green, P. Griffiths, and M. Kerr. Some enumerative global properties of variations of Hodge structures. Mosc. Math. J., 9(3):469-530, 2009.

[Gri68] P. Griffiths. Periods of integrals on algebraic manifolds. ii: Local study of the period mapping. American Journal of Mathematics, 90(54):805-865, 1968.

[Gri84] P. Griffiths. Topics in Transcendental Algebraic Geometry. Princeton University Press, 1984.

[GS69] P. Griffiths and W. Schmid. Locally homogeneous complex manifolds. Acta Mathematica, 123(1):253-302, 1969 .

[Hun90] B. Hunt. A bound on the Euler number for certain Calabi-Yau 3-folds. Journal für die reine und angewandte Mathematik, 411:137-170, 1990.

[KK98] V.S. Kulikov and P.F. Kurchanov. Algebraic Geometry III: Complex Algebraic Varieties Algebraic Curves and Their Jacobians. Springer, 1998.

[Lan73] A. Landman. On the Picard-Lefschetz transformation for algebraic manifolds acquiring general singularities. Transactions of the American Mathematical Society, 181(JUL):89126, 1973.

[Liu96a] K. Liu. Geometric height inequalities. Mathematical Research Letters, 3(5):693-702, 1996.

[Liu96b] K. Liu. Remarks on the geometry of moduli spaces. Proceedings of the American Mathematical Society, 124(3):689-695, 1996.

[LS04] Z. Lu and X. Sun. Weil-Petersson geometry on moduli space of polarized Calabi-Yau manifolds. Journal of the Institute of Mathematics of Jussieu, 3(2):185-229, 2004.

[Lu01] Z. Lu. On the Hodge metric of the universal deformation space of Calabi-Yau threefolds. Journal of Geometric Analysis, 11(1):103-118, 2001.

[M7̈8] W. Müller. Analytic torsion and R-torsion of Riemannian manifolds. Advances in Mathematics, 28(3):233-305, 1978.

[Pet00] C. Peters. Arakelov-type inequalities for Hodge bundles. arXiv:math/0007102v1 [math.AG], 2000.

[RS71] D. B. Ray and I. M. Singer. R-torsion and the Laplacian on Riemannian manifolds. Advances in Mathematics, 7(2):145-210, 1971.

[RS73] D. B. Ray and I. M. Singer. Analytic torsion for complex manifolds. Annals of Mathematics, 98(1):154-177, 1973.

[Sch73] W. Schmid. Variation of Hodge structure: The singularities of the period mapping. Inventiones mathematicae, 22(3):211-319, 1973.

[Tia87] G. Tian. Smoothness of the universal deformation space of compact Calabi-Yau manifolds and its Peterson-Weil metric. Mathematical Aspects of String Theory, pages 629-646, 1987.

[Tod89] A. N. Todorov. The Weil-Petersson geometry of the moduli space of SU( $\geq 3$ ) (Calabi-Yau) manifolds i. Communications in Mathematical Physics, 126(2):325-346, 1989. 
[TZ13] V. Tosatti and Y. Zhang. Triviality of fibered Calabi-Yau manifolds without singular fibers. Mathematical Research Letters, 21(4):905-918, 2013.

[Vie95] E. Viehweg. Quasi-projective Moduli for Polarized Manifolds. Springer, 1995.

[VZ05] E. Viehweg and K. Zuo. Numerical bounds for semi-stable families of curves or of certain higher-dimensional manifolds. Journal of Algebraic Geometry, 15(4):771-791, 2005.

[Wan03] C. Wang. Curvature properties of the Calabi-Yau moduli. Documenta Mathematica, 8:577-590, 2003.

[Yau78a] S.-T. Yau. A general Schwarz lemma for Kähler manifolds. American Journal of Mathematics, 100(1):197-203, 1978.

[Yau78b] S.-T. Yau. On the Ricci curvature of a compact Kähler manifold and the complex MongeAmpére equation, i. Communications on Pure and Applied Mathematics, 31(3):339-411, 1978.

[Yos] K.-I. Yoshikawa. Analytic torsion for Borcea-Voisin threefolds. arXiv:1410.0212v2.

[Yos14] K.-I. Yoshikawa. Degenerations of Calabi-Yau threefolds and BCOV invariants. International Journal of Mathematics, 26(4):217-250, 2014.

Kefeng Liu, Department of Mathematics, Capital Normal University, Beijing, 100048, China

Department of Mathematics, University of California at Los Angeles, California 90095

E-mail address: liu@math.ucla.edu

Wei Xia, Center of Mathematical Sciences, Zhejiang University, Hangzhou, 310027, CHINA.

E-mail address: xiaweiwei3@126.com 